\title{
Ten years of multi-wavelength follow-up observations of ANTARES neutrino alerts
}

The ANTARES ${ }^{\ddagger *}$, MWA, MASTER, ROTSE, Swift, TAROT and ZADKO Collaborations.

\# http://antares.in2p3.fr/Collaboration/index2.html

E-mail: dornicecppm.in2p3.fr

\begin{abstract}
High-energy neutrinos could be produced in the interaction of charged cosmic rays with matter or radiation surrounding astrophysical sources. To look for transient sources associated with a neutrino emission, a follow-up program of neutrino alerts is operating within the ANTARES Collaboration since 2009. This program triggers robotic optical telescopes (MASTER, TAROT, ROTSE, ZADKO and the SVOM ground based telescopes) immediately after the detection of each relevant neutrino candidate and schedules several observations in the following weeks. The most interesting neutrino candidates are also followed by the Swift and the INTEGRAL satellites, the Murchison Wide field Array radio telescope and the H.E.S.S. high-energy gamma-ray telescope. The results of ten years of observations are reported. No optical counterpart associated with a neutrino emission has been identified by ANTARES during image analysis and constrains on transient neutrino emission have been set.
\end{abstract}

Corresponding authors: D. Dornic ${ }^{\dagger 1}$, M.Ageron ${ }^{1}$, V. Bertin ${ }^{1}$, J. Brunner ${ }^{1}$, A. Coleiro ${ }^{2}$, F. Schüssler $^{3}$, D. Turpin ${ }^{1,4}$, B. Vallage ${ }^{2,3}$

${ }^{1}$ Aix Marseille Univ, CNRS/IN2P3, CPPM, Marseille, France.

2 APC, Univ Paris Diderot, CNRS/IN2P3, CEA/Irfu, Obs de Paris, Sorbonne Paris Cité, France.

${ }^{3}$ IRFU, CEA, Université Paris-Saclay, 91191 Gif-sur-Yvette, France.

${ }^{4}$ National Astronomical Observatories/Chinese Academy of Science 20A Datun Road, Beijing, 100012, China.

36th International Cosmic Ray Conference -ICRC2019-

July 24th - August 1st, 2019

Madison, WI, U.S.A.

\footnotetext{
*for collaboration list see PoS(ICRC2019)1177

† Speaker.
} 


\section{Introduction}

Doing astronomy with neutrinos is a long-standing dream for neutrino telescopes around the world. Up to now, IceCube [1] and ANTARES [2] are the key players for neutrino astronomy analyses. For more than ten years, the ANTARES Collaboration has a very rich multi-messenger program including an alert sending system and several time/space correlation analyses with a large diversity of potential neutrino sources such as core-collapse supernovae, gamma-ray bursts, tidal disruption events, fast radio bursts, micro quasars, flaring blazars, compact object mergers, etc [3]. Due to the ANTARES geographic location and its good pointing resolution, these studies have competitive sensitivities for neutrino signals from a field of view complementing that of IceCube. A high-energy neutrino diffuse flux of cosmic origin has been identified by the IceCube telescope in 2012 [4] and confirmed later with all neutrino flavours [5]. If confirmed in the future, the probable association between the neutrino alert IC170922 with a flare of the blazar TXS 0506+056 [6] will mark the real birth of the high-energy neutrino astronomy. ANTARES is also detecting a mild excess of neutrino events in both track and cascade channels at very high energies [7].

In this context, multi-messenger approaches consisting in simultaneously looking for the same sources with both neutrino telescopes, gravitational wave interferometers and/or multi-wavelength facilities constitute a privileged way of locating high-energy neutrino and cosmic ray sources and thus further understanding the acceleration mechanisms at play in these sources. An alert system, dubbed TAToO (Telescopes-Antares Target of Opportunity), has been operating since 2009 [8], sending regular alerts to our partners: the robotic telescopes of TAROT [9], ROTSE [10], ZADKO [11], MASTER [12], the SVOM ground telescopes [13], the X-ray telescopes of the Neil Gehrels Swift Observatory [14] and of INTEGRAL [15], the radio telescope Murchison Wide-field Array [16] and the very high energy H.E.S.S. [17] gamma-ray facility. During more than ten years of operations, we have built a very strong collaboration with our partners who followed a larger number of ANTARES neutrino alerts. This program is clearly a proof of the mutual benefits to look for the same sources at the same time with different messengers. The rapid provision of alerts for interesting neutrino events will enable both ground and space based observatories to quickly point at the direction of the alert. This fast follow-up will be vital to catch and characterise any multimessenger and multi-wavelength counterparts of these cataclysmic but shortlived phenomena such as gamma-ray bursts, fast radio bursts, supernova shock breakouts, etc.

The document is organized as followed: Section 2 describes the alert sending system and its performances. Follow-up observations of neutrino candidates are now performed over a broad range of the electromagnetic spectrum, from visible to gamma-ray. Section 3 reports the main results of the visible and X-ray observations. Section 4 shows the results of the multi-wavelength follow-up from radio and VHE gamma-rays. Finally, Section 5 describes potential interesting sources found during the follow-up of neutrino candidates.

\section{ANTARES alert sending system}

All the ANTARES events are reconstructed in real-time by two independent algorithms [18, 19]. These algorithms use an idealized detector geometry, neglecting the dynamical positioning calibration (the positions of the optical modules are not corrected for displacements due to sea 
currents). This reconstruction and subsequent quality selections allow the rate of events to be reduced from few $\mathrm{Hz}$ down to few $\mathrm{mHz}$ removing almost all the huge downgoing atmospheric muon contribution. The duty cycle of TAToO is around $>95 \%$. From the remaining atmospheric neutrino sample, the selection of the neutrino candidates with an increased probability to be of cosmic origin is performed with four online neutrino trigger criteria currently implemented in the TAToO alert system [8]:

- High energy (HE) trigger: the detection of a single high energy neutrino $(<\mathrm{E}>>5 \mathrm{TeV})$.

- Very high energy (VHE) trigger: the detection of a single very high energy neutrino $(<\mathrm{E}>>$ $30 \mathrm{TeV})$.

- Directional: the detection of a single neutrino for which the direction points toward $\left(<0.4^{\circ}\right)$ a local galaxy $(<20 \mathrm{Mpc})$ in the GWGC catalogue [20].

- Doublet trigger: the detection of at least two neutrinos coming from similar directions $\left(<3^{\circ}\right)$ within a predefined time window (15 min).

Until now, no doublet trigger has been sent to the network. Figure 1 shows the distribution of the number of hits and the total amplitude of these hits for the 3 first trigger types. Based on these two variables, a p-value to be a very high energy event is built. The trigger conditions are inspired by the features expected from astrophysical sources and are tuned to comply with the alert rate to be sent to the telescope network. An agreement between ANTARES and the optical telescope collaborations allows a rate of around 25 alerts per year to be sent to each optical telescope, while an agreement to send 6 alerts per year to the Swift satellite have been accepted. Due to this reduced rate, only the VHE sample is sent to MWA radio, Swift and INTEGRAL X-ray/gamma-ray observations. A similar sub-sample is sent to the MWA telescope. The TAToO alert system is able to send alerts within few seconds (6-7 s) after the neutrino detection with an angular resolution at around $\sim 0.5^{\circ}$. Figure 2 left displays the distribution of the delays between the time of the neutrinos and the time of the associated alert message.

The wide field of view and the fast response of the robotic optical telescopes (images can be taken within less than $20 \mathrm{~s}$ after the neutrino detection) are well suited to the search for transient sources. For each alert, the optical observation strategy is composed of an early follow-up (within 24 hours after the neutrino detection), to search for fast transient sources such as GRB afterglows, complemented by several observations during the two following months, to detect for example the rising light curves of CCSN. Each observation is composed of series of optical images (with clear filter). To cover most of the error point spread function (PSF) of the ANTARES neutrino alerts, Swift/XRT is perfoming 4 tiles of $2 \mathrm{ks}$ exposure each $(\approx 72 \%$ is covered $)$

\section{Optical and X-ray follow-up}

Between mid 2009 and July 2019, a total of 311 alerts have been sent to robotic telescopes while 19 targets of opportunity have been sent to the XRT instrument on board the Swift satellite since mid-2013. The typical follow-up efficiency is around $70-80 \%$ for the network of robotic telescopes and for the Swift satellite. 

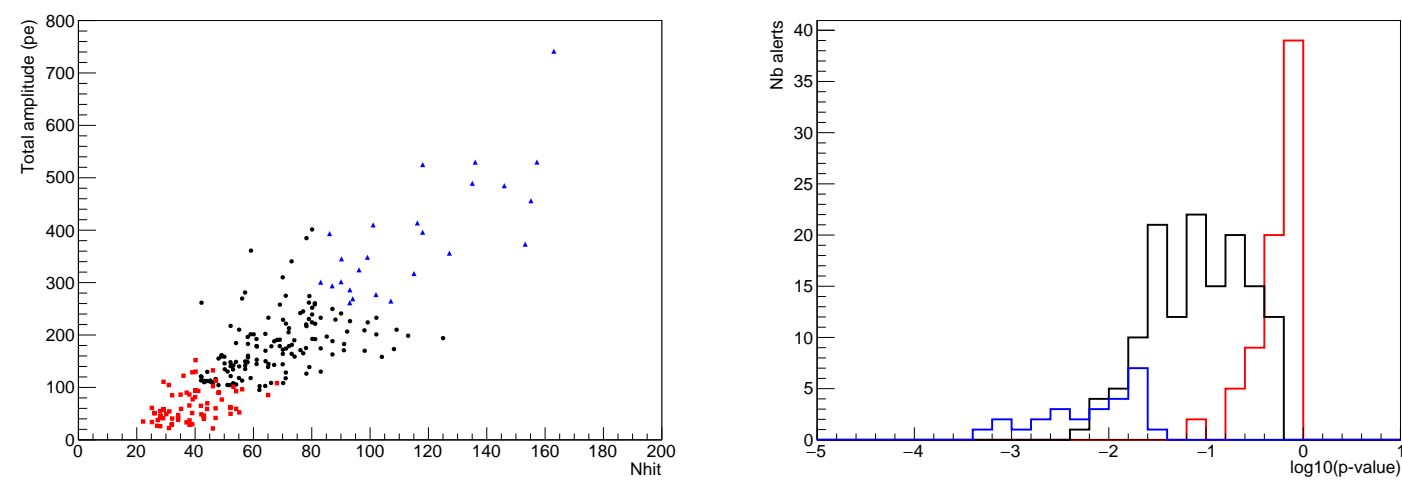

Figure 1: (Left) Distribution of the number of hits and the associated total amplitude of these hits for 20112018 alerts. The 3 colors correspond to the selection of events for each trigger: red points for Directional, black and blue points for the HE and VHE sample, respectivelly. (Right) Distribution of the p-values to be a VHE neutrino event for the types of triggers for 2011-2018 alerts.

From the 311 sent alerts, 208 triggers with early optical follow-up $(<24 \mathrm{~h}$ after the neutrino time) have been analyzed ( $\sim 67 \%$ of the sent alerts). Among them, 55 have a delay lower than 1 $\min (\sim 18 \%)$. Figure 2 right shows the delay between the first image of the follow-up performed by the robotic telescopes of TAROT and MASTER and the time of the neutrino. No clear optical counterparts were found and upper limits on the R-band magnitude of a transient astrophysical source have been derived. These limits correspond to the limiting magnitude of images, which is the faintest signal that can be detected. As we are looking for rapidly-fading sources, the signal is supposed to be more important in the first image of the observation, so the upper limits are the limiting magnitude of each first image computed at $5 \sigma$ and corrected for Galactic extinction [21]. By comparing these upper limits with optical afterglow light curves of gamma-ray bursts (Figure 3 left), it becomes possible to reject a GRB association with each neutrino alert, in particular when the optical follow-up is performed within a few minutes after the neutrino trigger [22]. A similar analysis has been carried out with Swift-XRT follow-ups of 19 ANTARES alerts [22] (Figure 3 right). The typical delay of the first Swift observation is around $6 \mathrm{~h}$ with a minimum delay of $1.1 \mathrm{~h}$. The probability to reject the GRB hypothesis reaches more than $\sim 70 \%$ if the X-ray followup occurs within few hours after the trigger (mainly dominated by the limited coverage of the ANTARES PSF with the 4 tiles).

Among the 311 sent alerts, 215 have sufficiently good optical long-term follow-ups, i.e. at least 3 (2) nights of observation for TAROT (MASTER) network. Among these 215 alerts, 74 were triggered by the directional trigger and 141 by the single HE/VHE triggers. No significant slowly varying transient optical counterparts were found in association with a neutrino trigger. The expected number of accidental SN detections, i.e. a SN detection in coincidence with a background neutrino event, is estimated to 0.4 in the 215 alerts assuming a rate of $1 \mathrm{SN}$ per year within a sphere of $10 \mathrm{Mpc}$ (i.e. $2.4 \times 10^{-4} \mathrm{yr}^{-1} \mathrm{Mpc}^{-3}$ ). This result is consistent with this small expectation $\mathrm{SN}$ number with a probability of $\approx 0.7$. 

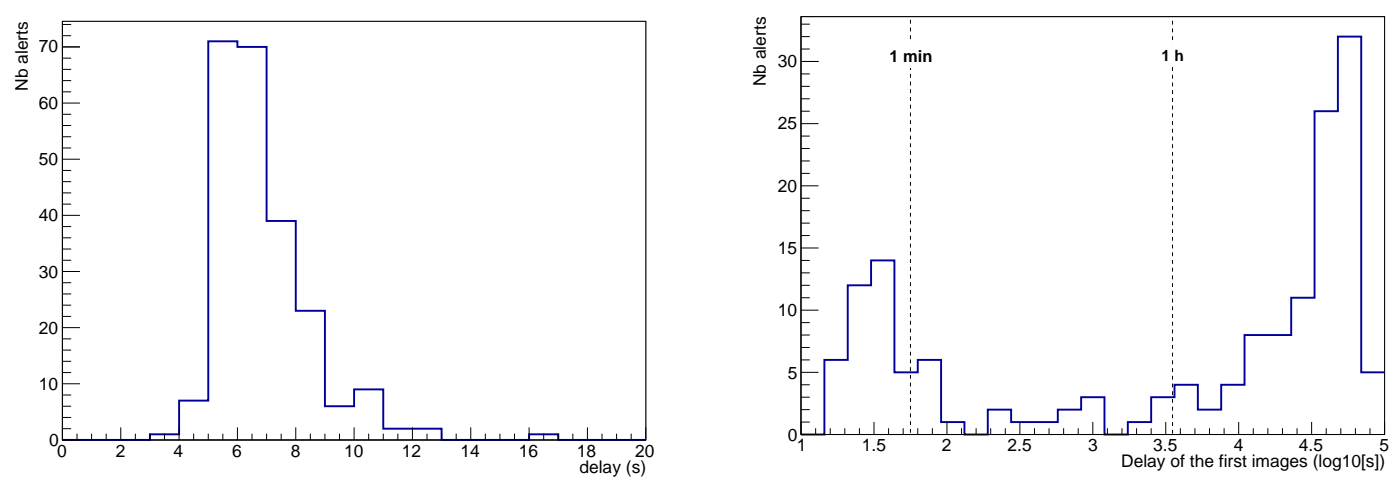

Figure 2: (Left) Distribution of the delays for 2011-2019 alerts between the time of the neutrino and the corresponding alert sending. (Right) Delay between the earliest images of the follow-up and the time of the neutrino for all the ANTARES alerts from 2011 and 2019.
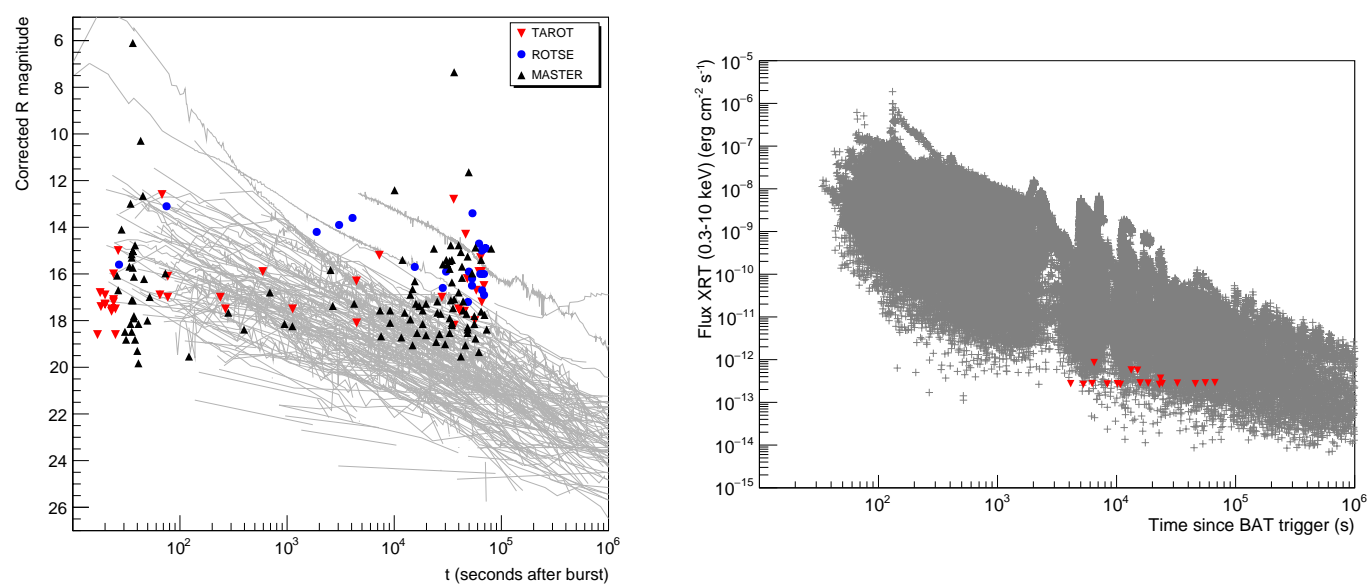

Figure 3: (Left) Comparison between archived optical light-curves for 301 GRBs detected in the period 1997-2014 and the upper limits obtained for the 208 neutrino alerts in the 2009-2019 period. Red, blue and black markers indicate upper limits on GRB afterglow magnitudes for neutrino alerts observed by TAROT, ROTSE and MASTER respectively. (Right) Comparison between 979 X-ray light-curves detected in the period 1997-2017 by Swift and the upper limits obtained for the 18 followed neutrino alerts in the 20132019 period (red markers).

\section{Radio and very high energy follow-up}

For the highest energy events, the follow-up can be extended to radio and VHE gamma-ray triggered observations. Up to now, four alerts have been followed by the Murchison Widefield Array (MWA) and two by the H.E.S.S. gamma-ray Cerenkov telescope. The results of the follow-up of ANT131121A and ANT140323A (2 directional triggers) by the MWA have been published in [23]. Several galaxies match the direction of the neutrinos in each case: NGC1374 and ESO358015 for ANT131121A, and ESO499-037 and PGC29194 for ANT140323A. Both neutrino events 
also had optical follow-ups with TAROT and ROTSE telescopes. No optical transient were identified in the error box of the two neutrinos. Transient or strongly variable radio sources consistent with the neutrino positions are looked for using MWA archival data at frequencies between 118 and $182 \mathrm{MHz}$, taken 20 days prior to, at the same time as, and up to a year after the neutrino triggers. As no radio counterparts are detected, $5 \sigma$ upper limits have been computed for low-frequency radio emission of $\sim 10^{37} \mathrm{erg} \mathrm{s}^{-1}$ for progenitors at $20 \mathrm{Mpc}$. If the neutrino sources are instead not in nearby galaxies, but originate in binary neutron star coalescences, the limits constrain the progenitors to be at $\mathrm{z} \geq 0.2$. The analyses of the two others neutrino alerts are in progress.

The very high gamma-ray H.E.S.S. telescopes have followed two ANTARES alerts shortly after the neutrino detection: ANT150901A and ANT170130A. For ANT150901A, the observations started on September 3rd, 2015, at 18:58 UT as soon as the necessary observation conditions were reached. No very-high energy gamma-ray source has been identified in the $1.5 \mathrm{~h}$ observations. This results an upper limit on the gamma-ray flux is set at $\Phi(\mathrm{E}>320 \mathrm{GeV} ; 99 \%$ C.L. $)<2.710^{-8} \mathrm{~m}^{-2}$ $\mathrm{s}^{-1}$ [24]. The neutrino ANT170130A direction has also been followed by H.E.S.S. with a very short delay of $32 \mathrm{~s}$ during 1 hour and again $45 \mathrm{~min}$ the night after. The offline analysis shows no source detection in the neutrino field of view [25].

\section{ANT150901A and ANT190410A}

These two neutrinos have been triggered with the VHE trigger and the main characteristics of these events are summarized in Table 1. For ANT150901A, Swift and MASTER have started the follow-up 9 and 10 hours after the neutrino triggers. The observation delays for ANT190410A are 13.1 and 9.2 hours for Swift and MASTER respectively. To characterize these transients, additional targets of oppportunity (ToO) have been requested to Swift during the following days after the triggers. In the follow-ups of both ANT150901A and ANT190410A, Swift/XRT has identified one uncatalogued, relatively bright and variable X-ray source above the Rosat All-Sky Survey (RASS) limit [26] $\left(=5 \times 10^{-13}-1.4 \times 10^{-12} \mathrm{erg} \mathrm{cm}^{-2} \mathrm{~s}^{-1}\right.$ at $\left.0.3-10 \mathrm{keV}\right)$ in the neutrino error box. Figure 4 shows the X-ray light curves observed for these two events. In the two cases, MASTER has identified a potential optical source but without significant flux variation.

\begin{tabular}{|c|c|c|c|c|c|}
\hline Name & Date & Ra, Dec & Nhit & Amplitude (pe) & p-value \\
\hline ANT150901A & $2015-09-0107: 38: 25$ & $246.31^{\circ},-27.47^{\circ}$ & 127 & 356 & 0.002 \\
ANT190410A & $2019-04-1013: 52: 04$ & $50.35^{\circ},-48.08^{\circ}$ & 82 & 400 & 0.030 \\
\hline
\end{tabular}

Table 1: Characteristics of the two neutrinos ANT150901A and ANT190410A.

For ANT150901A, a GCN notice [27] and an ATeL telegram [28] were emitted to request for more multi-wavelenght observations to characterize the star identified by MASTER (USNOB1.0 0626-0501169) and to test the association between the X-ray flare and this bright star. 19 multi-wavelength observatories have answered to this trigger covering the full electro-magnetic spectrum: 1 radio telescope, 11 optical/IR telescopes, 4 X-ray satellites and 4 very high-energy gamma-ray observatories. These observations point to USNO-B1.0 0626-0501169 being a young accreting G-K star or a binary system of chromospheric active stars (RS CVn), undergoing a flaring 
episode that produced the X-ray emission. The typical characteristics of this star are a distance of around $100-150 \mathrm{pc}$, an age of with a mass around $0.75 \mathrm{M}_{\odot}$. Therefore, this source seems not to be the origin of the bright ANT150901A neutrino with a probability of $\sim 3 \%$ of chance association. For ANT190410A, no public notice has been sent and only the information by MASTER, Swift and INTEGRAL are available. It seems that the neutrino is not associated to the transient. The complete analysis is still in progress for this event.
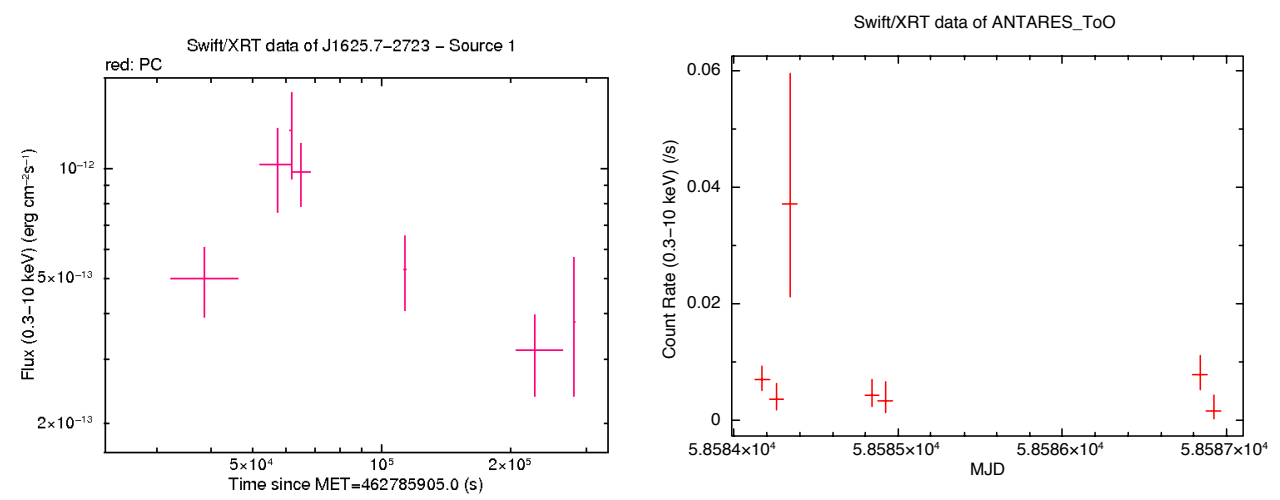

Figure 4: Light curves mesured by the XRT on board of Swift for the X-ray source identified in the followup of ANT150901A (left) and ANT190410 (right).

\section{Summary}

In this paper, we have summarized more than ten years of follow-ups of the ANTARES highenergy neutrino alerts. It is a very promising method to identify transient sources as sources of neutrino production as the detection of one counterpart associated in time and direction with one neutrino could lead to a high significance discovery. ANTARES is able to send alerts to the external community in $\sim 6$ seconds after the time of the neutrino detection with a precision of the direction better than 0.5 degrees. The triggers are followed by several multi-wavelength facilities such as robotic telescopes located all around the world, a radio telescope, two X-ray/gamma-ray satellites and very high-energy gamma-ray telescopes. More than 300 alerts have been sent and no counterpart has been detected in the different searches. This online analysis will be stopped mid 2020 when ANTARES will be decomissioned. A similar program is being implemented in the 2nd generation neutrino telescope in the Mediterannean Sea, KM3NeT [29], with largely improved performances.

\section{Aknowledgements}

The ANTARES Collaboration would like to take the opportunity of this proceeding to warmly thank the EM teams for their huge and constant effort on following its neutrino alerts and on having constructive discussions. 


\section{References}

[1] M.G. Aartsen et al, JINST 12 (2017) P03012.

[2] M. Ageron et al (ANTARES Collaboration), Nucl. Instr. Meth. A, 656, 11-38, 2011.

[3] D. Dornic et al, Pos(ICRC2019)872.

[4] Aartsen, M., Abbasi, R., Abdou, Y., et al. 2013a, PhRvL, 111, 021103; Aartsen, M., Abbasi, R., Abdou, Y., et al. 2013b, Sci, 342, 1242856.

[5] Aartsen M. G. et al. (IceCube Collaboration), ApJ, 809(1), 98 (2015).

[6] M.G. Aartsen et al, IceCube Collaboration, and other Collaborations, Science361 (2018), no. 6398, eaat 1378 .

[7] Albert A. et al. 2018a, ApJL, 853, L7; Fusco L. et al., Pos(ICRC2019)891.

[8] M. Ageron et al (ANTARES Collaboration), Astrpart. Phys., 35, 530-536, 2012.

[9] A. Klotz, M; Boer, J.-L. Atteia, B. Gendre, AJ, 137, 4100-4108, 2009.

[10] C.W. Akerlof et ak, PASP, 115, 132-140, 2003.

[11] D.M. Coward et al, 2016, PASA, 34, 5.

[12] V. Lipunov et al, Adv. in Astron., 30, 2010.

[13] Wei J., Cordier B et al (SVOM Collaboration), 2016, arXiv:1610.06892.

[14] D.N. Burrows et al, Space Sci. Rev., 120, 165-195, 2005.

[15] von Kienlin, A., Beckmann, V., Rau, A., et al. 2003, A\&A, 411, L299

[16] C.J. Lonsdale, R.J. Capallo, M.F. Morales et al , 2009 IEEE proceedings, 97. 1497. S.J. Tingay, R. Gaeker, J.D. Bowman et al, 2013, PASA, 32, 25.

[17] F. Aharonian et al (H.E.S.S. Collaboration), A\&A 457 (2006) 899.

[18] J.A. Aguilar et al (ANTARES Collaboration), Astropart. Phys., 34, 652-662, 2011.

[19] S. Adrián-Martínez et al (ANTARES Collaboration), Astrophys. J. Lett., 743, 14-19, 2011.

[20] D.J. White, E.J. Daw, V.S. Dhillon, Classical and Quantum Gravity, 28(8):080516, 2011.

[21] D.J. Schlegel, D.P. Finkbeiner, M. Dabvis, ApJ, 500, 525-553, 1998.

[22] S. Adrián-Martínez et al (ANTARES Collaboration), 2016, JCAP, 02, 062.

[23] S. Croft et al (MWA \& ANTARES Collaborations), The Astrophys. J. 823 (2016) no.2; L24.

[24] F. Schüssler, Proceedings of the Rencontres de Moriond 2017 (VHEPU), arXiv: 1705.08258.

[25] F. Schüssler et al, Pos(ICRC2017)253.

[26] W. Vogues et ak, A\&A, 349, 389-405, 1999.

[27] D. Dornic et al (ANTARES Collaboration), GCN, 18231 (2015).

[28] D. Dornic et al (ANTARES Collaboration), ATeL, 7987 (2015).

[29] S. Adrián-Martínez et al, (KM3NET Collaboration), Journal of Physics G: Nuclear and Particle Physics, 43 (8), 084001, 2016; R. Coniglione et al, Pos(ICRC2019)006. 